\title{
Cued aversive classical conditioning in humans: The role of trait-anxiety
}

\author{
Óscar Andión ${ }^{1,2,3^{*}}$, Xavier Caseras ${ }^{1,4}$, Miquel Àngel Fullana ${ }^{1}$, Alberto Fernandez-Teruel ${ }^{1}$, \\ Marc Ferrer $^{1,2}$, Miquel Casas ${ }^{1,2}$, Rafael Torrubia ${ }^{1}$ \\ ${ }^{1}$ Departament de Psiquiatria i Medicina Legal, Institut de Neurociencies, Universitat Autonoma de Barcelona, Bellaterra, Spain \\ ${ }^{2}$ Servei de Psiquiatria, Hospital Vall d'Hebron, Barcelona, Spain \\ ${ }^{3}$ Institut de Recerca Hospital Vall d'Hebron (VHIR), Universitat Autònoma, Bellaterra, Spain \\ ${ }^{4}$ Department of Psychological Medicine, Cardiff University, Cardiff, UK \\ Email: ${ }^{*}$ oandion@,vhebron.net
}

Received 25 January 2013; revised 28 February 2013; accepted 9 March 2013

Copyright (C) 2013 Óscar Andión et al. This is an open access article distributed under the Creative Commons Attribution License, which permits unrestricted use, distribution, and reproduction in any medium, provided the original work is properly cited.

\begin{abstract}
No study so far has specifically addressed the influence of individual differences in trait-anxiety on aversive classical conditioning as indexed by the startle reflex response. We compared the startle reflex responses between participants classified as high $(n=25)$ and low $(n=26)$ in trait-anxiety while undergoing a single-cue aversive classical conditioning procedure. High trait-anxiety group showed a greater startle response to the CS relative to the ITI at the post-acquisition compared with the pre-acquisition phase. Low trait-anxiety group did not show such a clear pattern of conditioning, and results from this group seem to be concealed by differences in the startle responses to the CS and the ITI during the pre-acquisition phase. However, a post-hoc analysis in which such differences at pre-conditioning were removed showed no conditioning effects in low trait-anxiety participants. Taking together, these results suggest differences between high and low trait-anxiety groups in the acquisition of the CS-US association. However, further research should clarify the unexpected pattern of responses shown by low trait-anxiety group.
\end{abstract}

Keywords: Classical Conditioning; Startle Reflex; Trait-Anxiety; Cued Conditioning; Fear-Potentiated Startle

\section{INTRODUCTION}

Aversive classical conditioning is the process by which a neutral stimulus acquires the capacity to elicit a negative

\footnotetext{
"Corresponding author.
}

emotional response (i.e. becomes a conditioned stimulus, CS) following its repeated pairing with an aversive unconditioned stimulus (US). This learning process has been consistently reproduced in animal and human research and constitutes a very influential model for anxiety disorders [1-3].

An increasing number of studies combining aversive classical conditioning paradigms and startle reflex (SR) have been published lately in the field of anxiety and its disorders [4-7]. The SR is a cross-species involuntary response to abrupt and intense stimulation consisting of a rapid and sequential top-down muscle contraction. In humans and animals, this response is consistently amplified when elicited during fear/anxiety and therefore seems to be a valid marker of aversive states [8].

Aversive conditioning studies in human have mainly focused on conditioned fear to discrete cues. However, conditioning procedures can also generate anxiety states as a result of context conditioning. In aversive conditioning paradigms, in which a conditioned stimulus (CS) is paired with an aversive unconditioned stimulus (US), competing associations develop between the CS and the experimental context (i.e. diverse background stimuli that are present at the time of conditioning). Cued fear conditioning emerges when the aversive CS temporally predicts the occurrence of an aversive US, while contextual fear conditioning appears when the aversive stimuli are perceived as unpredictable.

As regards cue conditioning, two basic experimental paradigms have been employed [7]: single cue and differential. During single cue conditioning, a single CS is repeatedly paired with the US. In differential conditioning paradigms, two different CSs are used, one CS is paired with the US (i.e. $\mathrm{CS}+$ ) and another is not (i.e. 
CS-). However, although fear conditioning has long been considered a central mechanism in anxiety disorders, results of studies employing these paradigms are rather inconsistent. In a meta-analysis carried out by Lissek et al. [7] it was found that differences in cue conditioning between anxiety patients and controls primarily emerged from studies employing single cue paradigms. Anxious patients tend to show modest increases in both acquisition of fear learning and conditioned responding during extinction.

Individual differences in fear acquisition and extinction in classical conditioning paradigms could function as diatheses which could partly explain vulnerability to anxiety. Substantial evidence indicates that trait-anxiety or neuroticism are vulnerability factors to develop anxiety disorders [9], however, it remains to be established to what extent these personality characteristics could be associated to individual differences in fear conditioning.

Recently, it has been proposed that during aversive classical conditioning anxious, patients and anxiety-prone individuals show an increased facility to associate unspecific environmental cues to the US (i.e. context conditioning), but not an increased ability to create CS-US associations (i.e. cue conditioning) [8]. It is inferred from this that trait-anxiety does not affect cue conditioning, but only context conditioning. However, most of the studies in which this assumption is based have been conducted in clinical samples [10] and therefore are liable to clinical status confounds (e.g. comorbidity or treatment), or aimed to investigate more complex learning processes (e.g. conditioned inhibition) and therefore few conclusions can be drawn on the influence of traitanxiety on the direct association between CS and US [5].

Single cue fear conditioning paradigms are widely used in animal research [11], and have shown to be useful in the study of conditioning differences between rats selected by their extreme responses in anxiety-related tasks [12]. To the best of our knowledge, there are no studies that have investigated in humans the role of trait-anxiety in aversive conditioning using a single cue paradigm and the SR as a measure of conditioning in non-clinical individuals selected by their extreme scores in anxiety.

The present study aims to fill this gap in the literature of classical conditioning and SR in human participants. We were interested in testing the hypothesis that high trait-anxiety participants would show greater aversive cue classical conditioning (i.e. CS-US association) compared to low trait-anxiety participants. To this end, we used a single cue classical conditioning paradigm, where a cue is systematically paired with an US during the acquisition phase.

\section{METHOD}

\subsection{Participants}

Participants were selected from a pool of 371 female undergraduate university students, who completed the Spanish version of the trait-anxiety scale of the StateTrait-Anxiety Inventory (STAI-T) [13]. Individuals scoring above or below the higher and lower quartile (scores $\geq 32$ or $\leq 16$ ) in the STAI-T were invited to participate (n $=64)^{1}$. The STAI-T was completed again on the day of the experimental session to confirm the participants' allocation to the high or low anxiety groups. Eight participants from the high and two from the low anxiety groups were discarded because of a change in their group classification after the second administration of the questionnaire. Three participants (one high trait-anxiety and two low trait-anxiety) were also excluded from the analysis because they showed no detectable startle response (i.e. eyeblink amplitude lower than $5 \mu \mathrm{V}$ in the first three consecutive startle probes during the initial habituation phase). Therefore, the final sample included 51 participants (25 high and 26 low anxious) aged 18 - 33. Participants were paid $15 €$ for their participation in the study.

\subsection{Procedure}

After completing the STAI-T, participants were contacted via telephone, given a general description of the study and screened for exclusion criteria (i.e. presence of psychiatric or neurologic disorders, visual or auditory impairment, use of drugs or psychotropic medication). Those who accepted to participate were scheduled for the experimental session.

Upon arrival to the laboratory, detailed information on the experimental session was given to the participants and their written consent was obtained. No instruction regarding the CS-US contingency was given and participants were not told that the procedure involved different phases. They were told that the study aimed to investigate how our body responds to different sort of stimuli and that they would be presented with visual stimuli and an electrical shock. They were also told that a noise would be played through the headphones but that they should try to ignore the noise as it was not directly related with the experimental task. After that, the electrodes were attached, the intensity of the shock was calibrated and participants completed the STAI-state scale. After a 2-minute accommodation period the experimental task began. Once the task finished and the electrodes were removed, participants completed the STAI-T (sec-

${ }^{1}$ It is important to notice that each item of the Spanish version of the STAI is scored on a $0-3$ scale, instead of the $1-4$ scale in the original English version and therefore the Spanish version yields lower scores than the original scale. 
ond administration) and a short questionnaire regarding their awareness of the CS-US.

The experimental task included five phases and a 2-minute rest period ordered as follows: habituation, preacquisition, acquisition, rest period, habituation, and post-acquisition. The rest period and the second habituation were included in order to control for context conditioning [4]. The presentation of the startle probe during the same context in which the US was previously presented, but in the absence of any CS or US is meant to abolish/decrease context conditioning, which due to the characteristics of our paradigm (single-cue and very short rest period) was already expected to be minimal.

Both habituation phases were identical and included 6 startle probes separated by an ITI of $30-80 \mathrm{~s}$ occurring during a black screen.

The pre-acquisition, acquisition and post-acquisition phases were identical regarding the order and timing of the CS and the startle probes presentation. However, during the acquisition phase all the CSs were immediately followed by the US. Each of these three phases consisted of $12 \mathrm{CSs}$ and 12 startle probes (6 delivered during CSs and 6 during ITIs) presented in semi-random order with the only constraint that no more than 2 startle probe-CS pairs appeared consecutively and that no startle probe was delivered during the first CS of the phase. Startle probes were presented 3.5 - $7.5 \mathrm{~s}$ after CS onset. The CS was presented for $8 \mathrm{~s}$ with an interval between CSs of 30 - 50 s. Startle probes were set apart a minimum of $18 \mathrm{~s}$ and a maximum of $35 \mathrm{~s}$.

The CS consisted of a yellow square (10 cm side) presented onto a 14 " screen $1.5 \mathrm{~m}$ in front of the participant. The US was a $50 \mathrm{~ms}$ duration electric shock delivered through two electrodes placed on the inside part of the participants' left wrist. It was produced via a S48 stimulator (Grass Inst., S48), transformed to constant current by CCU1A (Grass Inst., CCU1A) and isolated by SIU5 (Grass Inst., SIU5). The intensity of the US was individually adjusted starting from $2.5 \mathrm{~mA}$ to a level that was rated as greater than " 5 " on a $0-10$ pain scale (where 0 meant "no pain at all" and 10 "the worst pain I have ever felt"). The shock intensity was kept constant along the experimental task. Eight participants in the low anxiety and thirteen in the high anxiety groups received electric shocks above $2.5 \mathrm{~mA}$ (maximum intensity $3.5 \mathrm{~mA}$ ).

The whole procedure was approved by the Ethics Committee at the Autonomous University of Barcelona.

\subsection{Startle Elicitation, Recording and Quantification}

The startle probe was a $50 \mathrm{~ms}$ duration $105 \mathrm{~dB}$ burst of white noise with a near instantaneous rise time presented binaurally through headphones.

The eyeblink component of the SR was electromyog- raphically (EMG) measured by means of two $0.5 \mathrm{~cm}$ diameter silver surface disc electrodes placed $1 \mathrm{~cm}$ below and $1 \mathrm{~cm}$ medial from the external canthus of the left eye, following the guidelines set by Blumenthal et al. [14]. The ground electrode was placed on the forehead. Impedance level was kept below $5 \mathrm{~K} \Omega$. Physiological responses were recorded using a Biopac 150 polygraph (BIOPAC Systems, Inc.).

Following Blumenthal et al. [14] guidelines, the EMG raw data was digitalized at $1000 \mathrm{~Hz}$ rate, a $50 \mathrm{~Hz}$ notch filter was used to eliminate $50 \mathrm{~Hz}$ interference, an online bandpass filter 21.9 - $500 \mathrm{~Hz}$ was applied to the raw signal that was then full-wave rectified and integrated at time constant 10 using AcqKnowledge v.3.9.0 (BIOPAC Systems, Inc.).

The eye blink amplitude was calculated as the difference between the peak amplitude detected within the 21 $120 \mathrm{~ms}$ time frame following the startle probe onset and the baseline value. The baseline value was calculated by taking the mean EMG amplitude during the $50 \mathrm{~ms}$ preceding the presentation of the startle probe. Trials with excessive EMG activity during the first $20 \mathrm{~ms}$ were rejected and coded as missing. The number of rejected trials was low $(\mathrm{n}=37 ; 1.51 \%)$ and was not different between groups, $\mathrm{t}(49)=1.08, \mathrm{p}>0.1$. Trials that did not reach peak within $95 \mathrm{~ms}$ of onset latency were given a peak amplitude value of $0 \mu \mathrm{V}$ and were included in the analysis.

\subsection{Data Analysis}

Demographic and subjective data were compared between groups by means of students' t-tests or chi-square when appropriate.

The effect of conditioning was evaluated comparing the SR magnitude during the CSs relative to the SR magnitude during the ITIs, between the pre-acquisition and post-acquisition phases. With this aim, the startle magnitude during the ITIs was subtracted from the startle magnitude during the CSs and this difference submitted to an ANOVA including one within-participants factor (Phase: pre-acquisition vs. post-acquisition), and one between participants factor (Trait-anxiety: low vs. high). When appropriate, reduced degrees of freedom (Geisser-Greenhouse) were used to counter violations of the sphericity assumption.

Because the presence of the shock during the acquisition phase could produce sensitization effects, additional analyses were carried out to investigate the occurrence of this phenomenon. Accordingly, an ANOVA was conducted including the mean of the startle responses during the last two CSs and ITI of the pre-acquisition phase and the startle responses during the acquisition phase divided in three blocks including also two stimuli each (CS or ITI). Therefore, this ANOVA included two within- 
participants factors (Block: pre-shock, acquisition_1, acquisition_2, acquisition_3; and Stimuli type: CS v ITI) and one between-participants factor (Trait-anxiety: low vs. high).

Startle reflex responses were analyzed using raw scores and the intra-participants $t$ transformation, both analyses yielding very similar results. Only the results from the $\mathrm{t}$ transformed startle response are reported here. During the $\mathrm{t}$ transformation, startle responses greater or equal to $3 \mathrm{SD}$ above the individual's mean were considered outliers and were not included in the $t$ transformed score calculations.

\section{RESULTS}

The two groups did not differ in age, subjective perception of pain associated to the shock, intensity of the shock received or awareness of the CS-US association. As expected, the two groups were different in their scores to the STAI-T and STAI-state scales (Table 1).

The ANOVA including the pre-acquisition and postacquisition startle responses resulted in a significant two-way interaction: Phase $\times$ Trait-anxiety $^{2}, \mathrm{~F}(1,49)=$ 4.57, $\mathrm{p}<0.05, \eta_{\mathrm{p}}^{2}=0.085$. Post-hoc comparisons between phases for each trait-anxiety group showed that whereas in the low trait-anxiety group differences were not significant $[\mathrm{t}(25)=0.76, \mathrm{p}>0.1]$, a greater CS-ITI startle magnitude during post-acquisition than during pre-acquisition was found in the high trait-anxiety group $[\mathrm{t}(24)=2.17, \mathrm{p}<0.05]$. Post-hoc comparisons between groups for each phase showed no differences during postacquisition $[\mathrm{t}(49)=0.29, \mathrm{p}>0.1]$ and an unexpected difference during pre-acquisition: low trait-anxiety group showed higher CS-ITI than high trait-anxiety group [ $\mathrm{t}(49)$ $=3.17, \mathrm{p}<0.05)$ ] (Figure 1).

In order to ascertain if the lack of conditioning observed in low trait-anxiety group could be due to the between-groups difference at pre-acquisition, data were reanalyzed excluding those participants of each group whose value of the CS-ITI difference during pre-acquisition was $0.5 \mathrm{SD}$ above or below the total sample mean of the CS-ITI differences. 16 and 11 participants remained in the high and low trait-anxiety groups respectively after the application of this exclusion criterion. The results indicated that between groups differences during pre-acquisition disappeared [high trait-anxiety group: Mean CS-ITI $=-1.06$; $\mathrm{SD}=3.17$; low trait-anxiety group: Mean CS-ITI $=0.16$; $\mathrm{SD}=2.48 ; \mathrm{t}(25)=1.12$, $\mathrm{p}>0.1$ ], whereas between groups differences during post-acquisition remained unchanged [high trait-anxiety group: Mean CS-ITI $=4.12 ; \mathrm{SD}=6.77$; low trait-anxiety group: Mean CS-ITI $=1.85 ; \mathrm{SD}=4.36 ; \mathrm{t}(25)=0.98$,

\footnotetext{
${ }^{2}$ Very similar results were obtained after including the STAI-state scale as a covariate in the analysis; Phase $\times$ Trait-anxiety, $\mathrm{F}(1,48)=3.86, \mathrm{p}$ $<0.05, \eta^{2}=0.07$.
}

Table 1. Between groups differences in age, subjective intensity of the electric shock, physical intensity of the electric shock, STAI trait and state scores, and frequency of contingency aware participants.

\begin{tabular}{|c|c|c|c|c|}
\hline & $\begin{array}{l}\text { Low trait-anxiety } \\
\text { group } \\
(\mathrm{n}=26)\end{array}$ & $\begin{array}{l}\text { High trait-anxiety } \\
\text { group } \\
(\mathrm{n}=25)\end{array}$ & & \\
\hline & Mean (SD) & Mean (SD) & $\mathrm{t}$ & $\mathrm{p}$ \\
\hline Age & $21.00(2.65)$ & $20.96(3.53)$ & 0.05 & 0.96 \\
\hline Subj. shock & $6.31(1.13)$ & $6.08(0.81)$ & 0.82 & 0.42 \\
\hline Shock intensity & $2.69(0.38)$ & $2.82(0.35)$ & 1.25 & 0.22 \\
\hline STAI-T* & $9.19(4.77)$ & $35.52(8.61)$ & 6.15 & $<0.001$ \\
\hline \multirow[t]{2}{*}{ STAI-state } & $9.91(4.78)$ & $21.72(8.58)$ & 13.58 & $<0.001$ \\
\hline & n (\%) & n (\%) & $\begin{array}{c}\chi^{2} \\
(\mathrm{df}=1)\end{array}$ & $\mathrm{p}$ \\
\hline Awareness & $16(61.5)$ & $16(64.0)$ & 0.03 & 0.86 \\
\hline
\end{tabular}

*Second administration of STAI-T. Subj. shock: subjective intensity of the shock assessed on a scale from 0 to 10 . Shock intensity: physical intensity of the shock expressed in mA. Awareness: contingency awareness about the CS-US association.

$\mathrm{p}>0.1]$. Moreover, as in the analysis with the whole sample, a significant difference between pre and post-acquisition phases for high trait-anxiety $[\mathrm{t}(15)=$ $3.00, \mathrm{p}<0.05$ ] but not for low trait-anxiety participants $[\mathrm{t}(10)=1.02, \mathrm{p}>0.1]$ was observed.

The ANOVA performed to investigate the potential confounding effects of response sensitization during the acquisition phase resulted in the significance of the two within-participants factors (i.e. Stimuli Type and Block), reflecting a general higher response during CS than ITI $\left[\mathrm{F}(1,49)=38.43, \mathrm{p}<0.001, \eta_{\mathrm{p}}^{2}=0.44\right]$, and differences along the blocks included in the analysis $[\mathrm{F}(3,147)=$ 8.95, $\left.\mathrm{p}<0.001, \eta_{\mathrm{p}}^{2}=0.15\right]$. In fact, the factor Block showed a significant quadratic trend showing a peak immediately after the earlier shocks $[\mathrm{F}(1,49)=10.82, \mathrm{p}<$ 0.05 ; Figure 2]. This trend was confirmed by contrasting the startle responses during the different blocks included in the analysis. Thus, a significantly higher startle response was observed in the block which included the first two shocks when it was compared to any of the other blocks (smaller $\mathrm{t}(50)=4.87, \mathrm{p}<0.001)$. This sensitization effect of the SR due to the first appearance of the shock attenuated during the following blocks, which were not different among them or from the pre-shock block (all $\mathrm{p}>0.1$ ).

Because the sensitization effect from the shock was attenuated after the first block of acquisition, we also investigated group differences in aversive conditioning by comparing the startle responses during CS and ITI between the pre-shock block and the mean of the last two blocks in the acquisition phase. Student t-tests showed that low trait-anxiety participants did not differ in their responses during either the CS or the ITI between the 


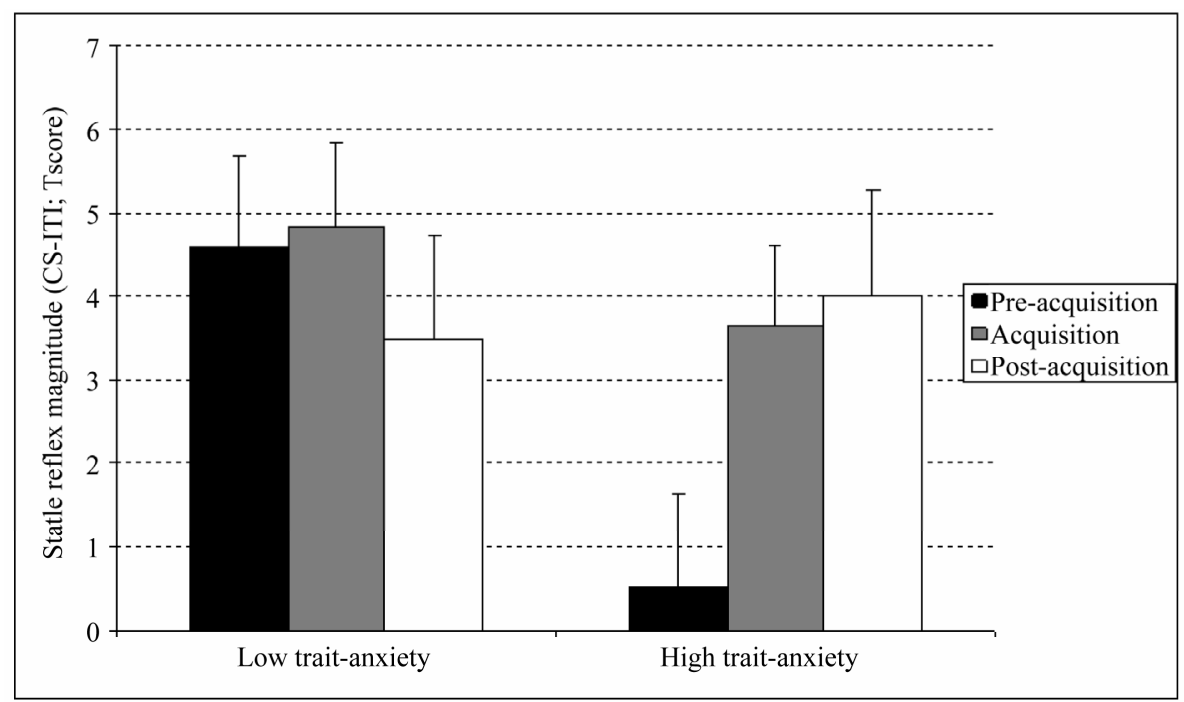

Figure 1. Mean CS-ITI t-score differences in startle magnitude during the experimental phases for high and low trait-anxiety groups. Error bars represent the standard error of the mean.

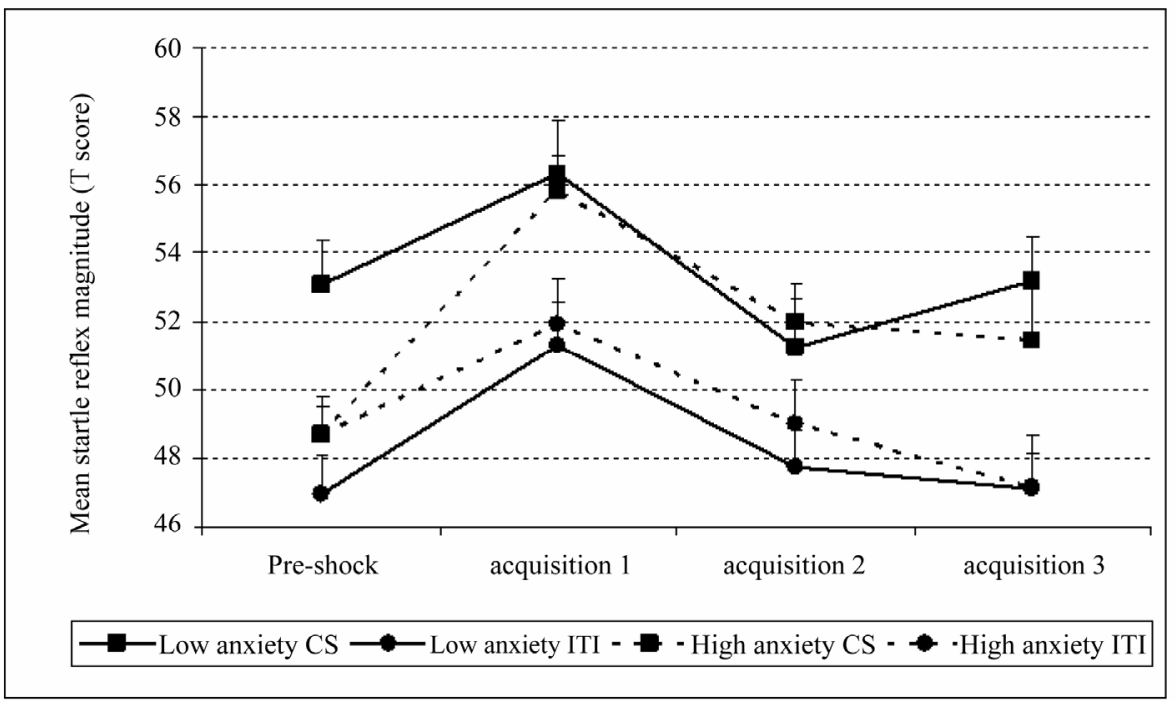

Figure 2. Mean startle response during the presentation of CS and ITI at the end of the preacquisition phase (pre-shock) and during the three blocs of the acquisition phase, separately for high and low trait-anxiety groups. Error bars represent the standard error.

pre-shock block and the latter blocks of the acquisition phase (all contrasts $p>0.1$ ). This was also true for high anxious participants regarding their responses during the ITI; however, the SR of this group during the CS was greater in the acquisition than in the pre-acquisition [ $\mathrm{t}(24)$ $=2.14, \mathrm{p}<0.05]$ (see Figure 2).

Finally, to test the effects of trait-anxiety on the potential occurrence of context conditioning, we compared the startle magnitudes during both habituation phases across trait-anxiety groups. The interaction Habituation Phase $\times$ Trait-anxiety was not significant, $\mathrm{F}(1,49)=0.009, \mathrm{p}>$ 0.1 , indicating no differences in the habituation slope between anxiety groups. The comparison of the SR during ITIs at pre- and post-acquisition across trait-anxiety groups revealed also no effect of trait-anxiety on context conditioning, Phase $\times$ Trait-anxiety $\mathrm{F}(1,49)=2.38, \mathrm{p}>$ 0.1 .

\section{DISCUSSION}

To our knowledge, this is the first study specifically designed to investigate aversive classical conditioning in a group of healthy volunteers selected by their extreme scores in trait-anxiety, using a single cue paradigm and the $\mathrm{SR}$ as a measure of conditioning. Our results suggest 
that there are differences in aversive classical conditioning between high and low trait-anxiety individuals: whereas the high trait-anxiety group showed clear cue conditioning effects, the low trait-anxiety group did not. An unexpected result was also found in the preconditioning phase: the low trait-anxiety group showed an increased startle magnitude during the CS relative to the ITI already during the pre-acquisition phase.

High trait-anxiety group showed an increase startle responses during the CS relative to the ITI at postacquisition when compared with pre-acquisition, indicating fear potentiated-startle to the cue after its association with the US. Conversely, low trait-anxiety group did not show differences in their startle response during the CS relative to the ITI between pre- and post-acquisition. Similarly, the analysis of conditioning during the acquisition phase also showed a different pattern of results for low and high trait-anxiety groups: the high trait-anxiety group showed a greater startle magnitude during CS but not during ITI at the acquisition compared to the pre-acquisition phase, but the low trait-anxiety group did not.

Despite that an increased general startle response to the CSs and the ITIs was observed during the first block of the acquisition, this sensitization effect dissipated in the following blocks of this phase in both groups. Thus, sensitization effects do not seem to explain our conditioning results neither when pre and post-acquisition phases were compared nor when pre-acquisition was compared with the last two blocks of the acquisition phase.

However, a closer look at the results of low traitanxiety participants shows a more complex picture. This group showed a greater startle response during the CS than the ITI already at the beginning of the task, even though no US had been yet delivered or instructions regarding its presentation given. Intriguingly, Grillon and Morgan [15] reported similar results using a differential classical conditioning paradigm, suggesting that these results might not be spurious and will require further investigation. Whereas the results of the high trait-anxiety participants are consistent with previous studies using single cue paradigms [7], those with the low anxiety group are not. At least two hypotheses for this unexpected finding are possible: a) participants in this group did not show fear conditioning; or b) because of the expectancies about the association between the CS and the shock generated by instructions at the beginning of the study, only this group began to show anticipatory responses during CS before to shock administration, and this response pattern remained until the post-acquisition phase.

As regards the first hypothesis, we reanalyzed our data excluding those participants of the two groups with the highest CS-ITI difference score at pre-acquisition. This strategy successfully removed the differences between groups at pre-acquisition, although the conditioning analyses showed identical results to those observed for the whole sample, i.e. a significant increase in the startle magnitude during CS relative to ITI after the CS-US pairing for high trait-anxiety group, and lack of any significant difference between pre- and post-acquisition for low trait-anxiety group. Our results are in line with Lissek's previous findings [7] suggesting that there are differences in aversive conditioning between anxious and non-anxious populations.

Despite that single cue paradigms have been successfully used in a number of studies in clinical and nonclinical samples, to our knowledge no study has been carried out using this paradigm in subjects selected by low trait-anxiety scores and changes in SR or skin conductance as dependent variables. Thus, it could be possible that low anxious subjects, a group which is not equivalent to the healthy control participants used in many clinical studies, were characterized by a heightened difficulty for the learning of excitatory aversive associations. This hypothesis is not incompatible with previous data, and two different kinds of evidence could indirectly support it, First, in the inbred Roman rat (i.e. RHA-I and RLA-I) strains, which have been psychogeneticallyselected for their extreme responses in anxiety-related tasks, the low anxious (and high impulsive) RHA-I strain do not show fear-induced potentiation of startle in a typical cue-conditioning procedure, while the relatively high anxious (and low impulsive) RLA-I strain show clear fear-potentiated startle responses [12]. Second, some seminal conditioning studies in psychopaths using single cue paradigms and electrodermal activity showed less conditioning in these subjects compared with control participants [16,17], and recent studies have also shown that psychopathic and antisocial individuals do not show increased skin conductance responses to $\mathrm{CS}+$ stimuli in differential conditioning paradigms $[18,19]$. Thus, antisocials, psychopaths and low trait-anxiety subjects could share an endophenotype related to vulnerability for a deficient excitatory conditioning to danger cues.

Regarding the second hypothesis, potentiation of startle response to the CS during pre-acquisition could be due to a higher expectancy of shock in low trait-anxiety group at this phase of the experiment despite that both groups had received the same instructions. If this was the case, it would mean that this group shows better associative learning. However to our knowledge no study has been specifically addressed to this aim and the published data do not confirm this hypothesis [7].

We did not find context conditioning in the present study. In fact, it was designed to control for any contextual potentiation of the SR that could have obscured the 
responses during post-acquisition. The fact that the startle responses during this second habituation phase were lower than the responses during the initial habituation, suggests that context conditioning did not occur.

A number of limitations of this study should be mentioned. Firstly, the present study only included female participants, which limits our capacity to extrapolate these results to the general population. Previous research showed gender-specific differences in aversive cue and context conditioning [20], these previous results highlight the importance of accounting for gender in future research. Secondly, the single cue conditioning paradigm used in the present study makes difficult to control for possible sensitization effects; the introduction of a control group would have made simpler the interpretation of our results. Finally, not including measures of subjective responses to the CS would have been helpful in the interpretation of the psychophysiological data.

To sum up, our results support the existence of differences in single cue aversive conditioning between high and low trait-anxiety participants. High trait-anxiety individuals show a clear pattern of startle potentiation during the CS after its pairing with the US whereas low trait-anxiety individuals do not. Future research including larger sample sizes, male and female participants, subjective responses to the CS and a control group should shed more light on the role of trait-anxiety in this conditioning paradigm.

\section{ACKNOWLEDGEMENTS}

Funded by the Spanish Ministry of Education and Science (ref. SEJ2006-10237) and the Catalan government (ref. 2009-SGR-51). A.F-T receives support from PSI2009-10532 and "La Marató de TV3" (ref 092630/31).

\section{REFERENCES}

[1] Rachman, S. (1977) The conditioning theory of fear-acquisition: A critical examination. Behaviour Research and Therapy, 15, 375-387. doi:10.1016/0005-7967(77)90041-9

[2] Marks, I. and Tobeña, A. (1990) Learning and unlearning fear: A clinical and evolutionary perspective. Neuroscience and Biobehavioral Reviews, 14, 365-384. doi:10.1016/S0149-7634(05)80059-4

[3] Ohman, A. and Mineka, S. (2001) Fear, phobias and preparedness: Towards and evolved module of fear and fear learning. Psychological Review, 108, 483-522. doi:10.1037/0033-295X.108.3.483

[4] Ameli, R., Ip, C. and Grillon, C. (2001) Contextual fearpotentiated startle conditioning in humans: Replication and extension. Psychophysiology, 38, 383-390. doi:10.1111/1469-8986.3830383

[5] Grillon, C. and Ameli, R. (2001) Conditioning inhibition of fear-potentiated startle and skin conductance in humans. Psychophysiology, 38, 807-815.

doi:10.1111/1469-8986.3850807

[6] Grillon, C., Cordova, J., Morgan, C.A., Charney, D.S. and Davis, M. (2004) Effects of the beta-blocker propranolol on cued and contextual fear contitioning in humans. Psychopharmacology, 175, 342-352. doi:10.1007/s00213-004-1819-5

[7] Lissek, S., Powers, A.S., McClure, E.B., Phelps, E.A., Woldehawariat, G., Crillon, C. and Pine, D.S. (2005) Classical fear conditioning in the anxiety disorders: A meta-analysis. Behaviour Research and Therapy, 43, 1391-1424. doi:10.1016/j.brat.2004.10.007

[8] Grillon, C. (2008) Models and mechanisms of anxiety: Evidence from startle studies. Psychopharmacology, 199, 421-437. doi:10.1007/s00213-007-1019-1

[9] Clark, L.A., Watson, D. and Mineka, S. (1994) Temperament, personality, and the mood and anxiety disorders. Journal of Abnormal Psychology, 103, 103-116. doi:10.1037/0021-843X.103.1.103

[10] Grillon, C. (2002) Startle reactivity and anxiety disorders: Aversive conditioning, context, and neurobiology. Biological Psychiatry, 52, 958-975. doi:10.1016/S0006-3223(02)01665-7

[11] Winslow, J.T., Noble, P.L. and Davis, M. (2007) Modulation of fear-potentiated startle and vocalizations in juvenile rhesus monkeys by morphine, diazepam, and buspirone. Biological Psychiatry, 61, 389-395.

doi:10.1016/j.biopsych.2006.03.012

[12] López-Aumatell, R., Blázquez, G., Gil, L., Aguilar, R., Cañete, T., Giménez-Llort, L., Tobeña, A. and Fernández-Teruel, A. (2009) The roman high- and low-avoidance rat strains differ in fear-potentiated startle and classical aversive conditioning. Psychothema, 21, 27-32.

[13] Spielberger, C.D., Gorsuch, R.L., Lushene, R., Vagg, P.R. and Jacobs, G.A. (1983) Manual for the state-trait anxiety inventory (Form Y self-evaluation questionnaire). Consulting Psychologist Press, Palo Alto.

[14] Blumenthal, T.D., Cuthbert, B.N., Filion, D.L., Hackley, S., Lipp, O.V. and van Boxtel, A. (2005) Committee report: Guidelines for human startle eyeblink electromyographic studies. Psychophysiology, 42, 1-15. doi:10.1111/j.1469-8986.2005.00271.x

[15] Grillon, C. and Morgan, C. A. 3rd. (1999) Fear-potentiated startle conditioning to explicit and contextual cues in Gulf War veterans with posttraumatic stress disorder. Journal of Abnormal Psychology, 108, 134-142. doi:10.1037/0021-843X.108.1.134

[16] Hare, R.D. (1965) Acquisition and generalization of a conditioned-fear response in psychopathic and nonpsychopathic criminals. Journal of Psychology, 59, 367-370. doi:10.1080/00223980.1965.10544625

[17] Lykken, D.T. (1957) A study of anxiety in the sociopathic personality. Journal of Abnormal Psychology, 55, 6-10. doi: $10.1037 / \mathrm{h} 0047232$

[18] Birbaumer, N., Veit, R., Lotze, M., Erb, M., Hermann, C., Grodd, W. and Flor, H. (2005) Deficient fear conditioning in psychopathy. A functional magnetic resonance 
imaging study. Archive of General Psychiatry, 62, 799805. doi:10.1001/archpsyc.62.7.799

[19] Gao, Y., Raine, A., Dawson, M.E. and Mednick, S.A. (2010) Association of poor childhood fear conditioning and adult crime. American Journal of Psychiatry, 167, 56-60. doi:10.1176/appi.ajp.2009.09040499
[20] Grillon, C., Dierker, L. and Merikangas, K.R. (1998) Fear-potentiated startle in adolescent offspring of parents with anxiety disorders. Biological Psychiatry, 44, 990997. doi:10.1016/S0006-3223(98)00188-7 